\title{
Synthesis, Anticonvulsant Activity and In silco Studies of Schiff Bases of 2-Aminothiophenes via Guanidine- Catalyzed Gewald Reaction
}

\author{
Pavan Kumar Kunda ${ }^{1^{*}}$, J Venkateswara Rao ${ }^{2}$, K Mukkanti ${ }^{3}$, \\ Madhusudhanareddy Induri ${ }^{4}$ and G Deepak Reddy ${ }^{5}$ \\ ${ }^{1}$ Department of Pharmaceutical Chemistry, CM College of Pharmacy, Maisammaguda, Dhulapally, Secunderabad, \\ ${ }^{2}$ Department of Pharmaceutical Chemistry \& Pharmaceutical Analysis, Sultan-ul-uloom College of Pharmacy, Banjara Hills, \\ ${ }^{3}$ Director IST, JNTUH, Hyderabad, ${ }^{4}$ Department of Pharmaceutical Chemistry, Malla Reddy College of Pharmacy, \\ Maisammaguda, Secunderabad, ${ }^{5}$ Research Division, Dept. of Medicinal Chemistry, Vishnu Institute of Pharmaceutical \\ Education and Research, Narsapur, Andhra Pradesh, India
}

*For correspondence: Email: pavanreddy79@gmail.com; Tel: +91-9160044376

\begin{abstract}
Purpose: To synthesize Schiff bases of 2-aminothiophenes and evaluate their anticonvulsant activity and in silco properties

Methods: 2-Amino-N-o-tolyl-5,6-dihydro-4H-cylcopenta[b]thiophene-3-carboxamide was synthesized using 1,1,3,3-tetramethylguanidine lactate as a basic catalyst and by microwave irradiation. 2substitued-o-tolyl-5,6-dihyro-4H-cylcopenta[b]thiophene-3-carboxamide was prepared by reacting with different substituted aromatic aldehydes. The synthesized compounds were characterized by Fourier transform infrared spectroscopy (FTIR), nuclear magnetic resonance spectroscopy $\left(^{1} \mathrm{H} N M R\right)$ and mass spectrometry (MS) while their anticonvulsant activity was screened against maximum electroshockinduced seizure (MES), and pentylenetetrazole-induced seizure (PTZ) against phenytoin and diazepam as reference standards. Molecular docking (in silico) studies were performed using 4-aminobutyrateaminotransferase in order to predict possible protein-ligand interactions.

Results: Among the 21 synthesized compounds, $2 b, 2 d, 2 f, 2 k, 2 m, 2 n$ and 20 showed good to moderate activity against MES and PTZ-induced convulsions. Compounds $\mathbf{2 b}, \mathbf{2 d}, \mathbf{2} \boldsymbol{f}, \mathbf{2} \boldsymbol{k}$ and $\mathbf{2 m}$ exhibited lower activity against PTZ than against MES model while compounds $2 n$ and 20 afforded greater protection against PTZ than against MES model. In silico results also revealed maximum binding affinity to GABA-AT protein which was higher than other compounds

Conclusion: The synthesized compounds showed potent anticonvulsant activity. Molecular docking results should give an insight into how further modification of lead compound can be carried out for higher inhibitory activity.
\end{abstract}

Keywords: Ionic liquid, 2-Aminothiophenes, Anticonvulsant, In silco studies, Molecular docking.

Tropical Journal of Pharmaceutical Research is indexed by Science Citation Index (SciSearch), Scopus, International Pharmaceutical Abstract, Chemical Abstracts, Embase, Index Copernicus, EBSCO, African Index Medicus, JournalSeek, Journal Citation Reports/Science Edition, Directory of Open Access Journals (DOAJ), African Journal Online, Bioline International, Open-J-Gate and Pharmacy Abstracts

\section{INTRODUCTION}

Thiophene scaffold have a variety of biological activities such as antitubercular [1], antioxidant [2], anti-HIV-PR inhibitors [3], antimicrobial [4], multitargeted kinase inhibition [5], $A_{1}$ adenosine receptor allosteric enhancement [6]. Of Several approaches for the synthesis of 2aminothiophenes have been reported including the use of microwave assisted synthesis [7], synthesis from thiomorpholides [8], coupling reaction [9], solid support [10], base-induced inter- and intra-molecular C-S and C-C bond 
formation [11] and ultrasonication [12]. Ionic liquids (ILs) have attracted attention for green organic synthesis because of their unique physical and chemical properties such as nonvolatility, non-flammability, thermal stability, recyclability and controlled miscibility. Synthesis using ionic liquid has great advantages including easy workup, milder reaction and high yields. Molecular docking studies have been performed on synthesized compounds with gammaaminobutryic acid aminotransferase (GABA-AT) in order to identify possible binding conformations of the ligand. [13]. The aim of the present research was to study some Schiff bases for anticonvulsant activity and ligand docking with gamma-aminobutryic acid aminotransferase in order to identify possible binding conformations. By performing molecular docking studies, one can identify possible binding conformations of ligands, and the data obtained would be very helpful to achieve further modification of lead for greater inhibitory activity.

\section{EXPERIMENTAL}

Melting point was determined using an electrochemical thermal apparatus (IA 9100 Digital melting point apparatus) and were uncorrected. FT-IR spectra were recorded on a Perkin Elmer FT-IR spectrometer. ${ }^{1} \mathrm{H} \quad \mathrm{NMR}$ spectra were obtained with Bruker Spectrometer (400 MHz) in $\mathrm{CDCl}_{3}$ ( deuterated chloroform) and DMSO (dimethyl sulfoxide) using tetramethylsilane (TMS) as an internal standard. Mass spectra were obtained with LC-MSD Trap-SL 2010 A Schimadzu. All the compounds were synthesized according to the scheme in Figure 1.

Synthesis of 2-cyano-N-o-tolylacetamide(1): A mixture of o-toluidine $(0.01 \mathrm{~mol})$ and ethylcyanoacetate $(0.655 \mathrm{~mol})$ was heated at $180^{\circ} \mathrm{C}$ in a microwave (Raga Scientific Microwave system model RG31L) at $2450 \mathrm{MHZ}$ for $5 \mathrm{~min}$. The reaction mixture was left at room temperature overnight. The solid obtained was separated, washed with ethanol, dried and recrystallized from acetone:water mixture.

Synthesis of 2-amino-N-o-tolyl-5,6-dihydro$4 \mathrm{H}$-cyclopenta[b]thiophene-3-carboxamide under microwave irradiation(2): $A$ mixture of cyclopentanone $(0.04 \mathrm{~mol}), \quad$ 2-cyano-N-otolylacetamide $(0.04 \mathrm{~mol})$, sulphur $(0.04 \mathrm{~mol})$, ionic liquid $(1.5 \mathrm{~g})$, ethanol $(15 \mathrm{ml})$ were placed in a volumetric flask and stirred for $5 \mathrm{~min}$. The reaction mixture was placed in a microwave at $100 \mathrm{~W}$ for $3 \mathrm{~min}$. The progress of the reaction was monitored by thin layer chromatography (TLC) Water $(10 \mathrm{ml})$ was added during which reddish brown crystals were separated. The preciptated product was filtered under suction. lonic liquid was recovered by removing water under reduced pressure stored in a desicator for its reuse.

General procedure for synthesis of 2substituted N-o-tolyl-5,6-dihydro-4H-cyclopenta[b]thiophene-3-carboxamide under conventional heating (2): A mixture of 2-amino$\mathrm{N}$-o-tolyl-5,6-dihydro-4H-cyclopenta[b]thiophene3-carboxamide $(0.02 \mathrm{~mol})$ and appropriately subtituted aryl aldehydes/ketones $(0.02 \mathrm{~mol})$ was dissolved in ethanol in the presence of catalytic amount of glacial acetic acid $(1 \mathrm{ml})$ by heating under reflux for $5 \mathrm{~h}$. The reaction mixture was cooled overnight, the separated solid was filtered washed with isopropyl alcohol and recrystallized

General procedure for synthesis of 2 substitutedN-o-tolyl-5,6-dihydro-4H cyclo penta[b]thiophene-3-carboxamide under microwave irradiation(2): A mixture of 2amino-N-o-tolyl-5,6-dihydro-4H-cyclopen-

ta[b]thiophene-3-carboxamide $(0.02 \mathrm{~mol})$ and appropriately subtituted aryl aldehydes/ketones $(0.02 \mathrm{~mol})$ was added to acidic alumina $(1 \mathrm{~g})$ at room temperature. The reaction mixture was mixed, allowed to adsorb on acidic alumina, dried and kept inside the alumina bath and irradtiated at $750 \mathrm{~W}$ for $30 \mathrm{~s}$. The reaction mixture was cooled, the product extracted with ethyl acetate, concentrated and cooled. The solid product was separated filtered, washed thoroughly with water and recrystallized from dimethyl formaamide.

\section{Evaluation of anticonvulsant activity}

Male albino Swiss mice, weighing $20-25 \mathrm{~g}$, were used to study the effect of the synthesized compounds on MES- and PTZ-induced seizures. Female animals were excluded because of the fact that estrous cycle could influence their activity threshold. They were procured from National Institution of Nutrition; Hyderabad and had free access to balanced food and water ad libitium Prior to experimentation, the animals were allowed a week time to acclimatize to laboratory conditions. However, food, but not water, was withdrawn $8 \mathrm{~h}$ before and during the experiments. 
$\mathrm{H}_{3} \mathrm{C}$<smiles>CCOC(=O)OC[NH2+]c1cccc(C)c1</smiles>

O-toluidine Ethylcyanoacetate<smiles>C=Nc1sc2c(c1C(=O)Nc1ccccc1C)CCC2</smiles>

$30 \mathrm{sec}$

2a $\mathrm{R}=\mathrm{H}$

2b R=3-OCH $\mathrm{CH}_{3}, 4-\mathrm{OH}$

2c $\mathrm{R}=4-\mathrm{CH}_{3}$

2d R=2- $-\mathrm{NO}_{2}$

2e $\mathrm{R}=3-\mathrm{NO}_{2}$

2f $\mathrm{R}=4-\mathrm{NO}_{2}$

$2 \mathrm{~g} \mathrm{R}=4 \mathrm{CH}\left(\mathrm{CH}_{3}\right)_{2}$

2h $\mathrm{R}=4 \mathrm{~F}$

2i $\mathrm{R}=4-\mathrm{N}\left(\mathrm{CH}_{3}\right)_{2}$

2j $\mathrm{R}=2,4-\mathrm{di}-\mathrm{cl}$

$2 \mathrm{k} \mathrm{R}=2-\mathrm{cl}$

2l $\mathrm{R}=2,4-\left(\mathrm{OCH}_{3}\right)_{2}$

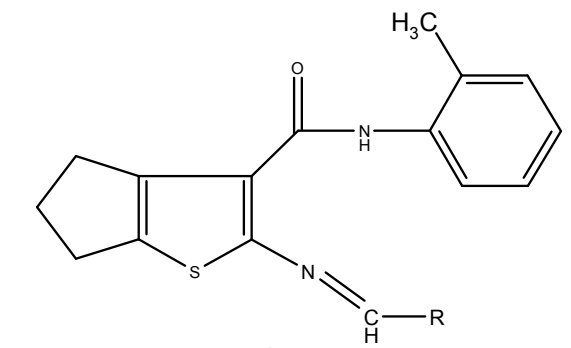

$2 \mathrm{~m} \quad \mathrm{R}=$<smiles>Cc1ccco1</smiles>

$2 n \quad R=$<smiles>Cc1c[nH]c2ccccc12</smiles>

$20 \mathrm{R}=$

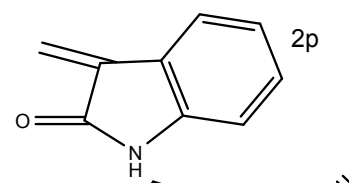

$2 q R=$

$2 s \mathrm{R}=$
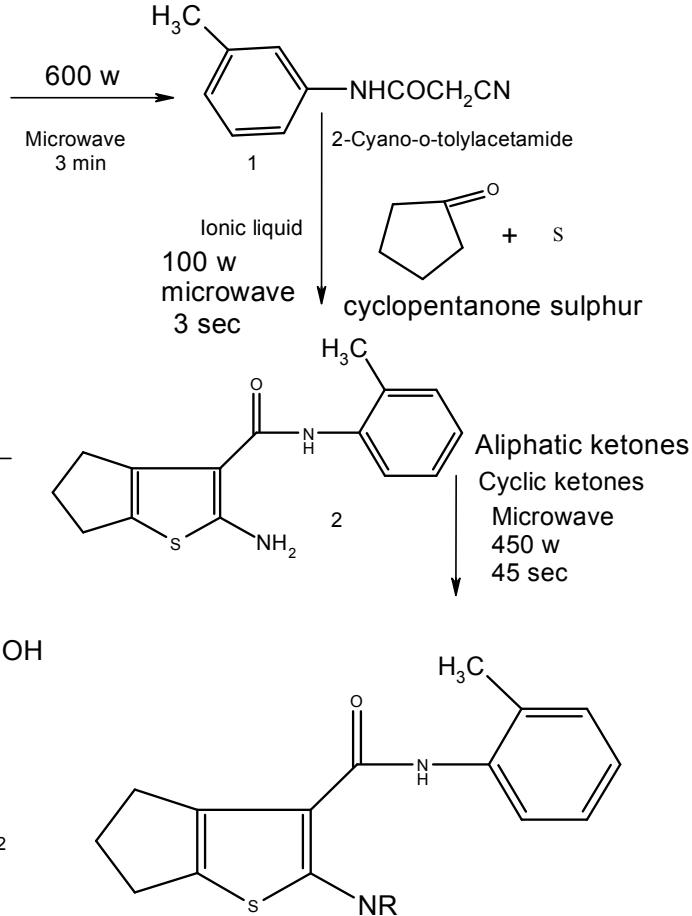

(1)

2t $R=$

Figure 1: Synthesis of 2-substituted- N- o-tolyl-5, 6-dihydro-4H-cyclopenta[b]thiophene-3-carboxamide

Animal experiments were performed in accordance with the institutional guidelines for animal experimentation[14]. Institutional animal ethical permission was obtained from Malla Reddy College of Pharmacy (ref no. $1217 / a / 08 / C P C S E A)$ which is affiliated to Osmania University, prior to the animal studies. All the protocols and the experiments were conducted in strict compliance according to ethical principles and guidelines the animals received due care according to the criteria outlined in the "Guide for the Care and Supervision of Experiments on Animals" prepared of the National Academy of Sciences and published by National Institutes of Health, USA.

\section{Maximal electroshock-induced seizure test}

Seizure was induced by maximal electroshock in albino mice $(n=6)$ with the aid of an electroconvulsiometer (EC 01,Orchid Scientifics) by passing a current of $45 \mathrm{~mA}$ for $0.2 \mathrm{~s}$ using ear clip electrodes. The duration of different phases of seizures was noted [15].

\section{Pentylenetetrazole-induced seizure test}

Trop J Pharm Res, August 2013;12 (4): 
Albino mice $(n=6)$ were treated with test prior to subcutaneous injection of PTZ $(80 \mathrm{mg} / \mathrm{kg})$, The mice were observed for clonic and tonic convulsions and $24 \mathrm{hr}$ mortality. The mice were observed for onset of convulsion up to $30 \mathrm{~min}$ after PTZ administration.

\section{In silco studies}

A set of 21 different ligands were drawn using Chemsketch, (11.0 ACD labs) while geometrical optimization was carried out with LigPrep.2.5 (Schrodinger Suite 2011). Using this tool, a single low energy 3D structure was obtained for each ligand and different conformers obtained during ionization of the ligands using EPIK module which generates ionization states in the $\mathrm{pH}$ range of $7 \pm 2$. The molecular properties of the minimized ligands were studied using QIKPROP module 2.5 Schrodinger suite 2011 which predict absorption, distribution, metabolism and excretion (ADME, Quiprop version 3.4, Schrodinger Suite 2011) [16].

Ligand protein interaction of 2-aminosubstituted thiophenes with GABA-AT was studied by docking GLIDE-XP. The protein structure was retrieved from the protein data bank server (PDB entry 1OHV, (http://www.rcsb.org/pdb/home/ home.do). The structure of the protein was corrected by adding hydrogen and removing water moieties with force field OPLS-2005, i.e., optimized potentials for liquid simulations. The active site was identified by SITEMAP tool. Receptor grid generation was carried out using glide; the ligands were docked by employing XP mode and they were docked flexibly. Best pose was selected based on E-model energy and docking score from glide (glide score) was entirely based on Chem score. It also includes a steric-clash term, and adds polar terms featured by Schrödinger to correct electrostatic mismatches $[17,18]$.

\section{Statistical analysis}

The data are expressed as mean \pm standard error of mean (SEM). One-way analysis of variance (ANOVA) followed by Tukey multiple comparison test was carried out with Graph Pad Prism software (version 3.0). Differences between means were considered significant at $p$ $<0.01$.

\section{RESULTS}

Compound 2b with 4-hydroxy-3-methoxy substitution showed good activity compared to control and standard, while compounds $2 \mathrm{~m}, 2 \mathrm{n}$ and 20 with heterocyclic ring system such as furan, indole and isatin, showed moderate activity against maximum electroshock-induced seizure (MES). In the case of pentylene tetrazole-induced seizure, compounds $2 b, 2 e, 2 f$, 20 and $2 q$ with substitutions 4-Hydroxy-3methoxybenzylideneamino,3-nitrobenzylideneamino, 4-nitrobenzylideneamino, -(2-oxoindolin3-ylideneamino, 1-methylpyrrolidin-2-ylideneamino substitution at 2-amino-N-o-tolyl-5,6dihydro-4H-Cyclopenta[b]thiophene-3-carboxamide produced greater reduction in the duration of convulsion than control but lower than the reference standard. All the synthesized compounds showed lower protection against PTZ-induced seizure than diazepam (reference standard).

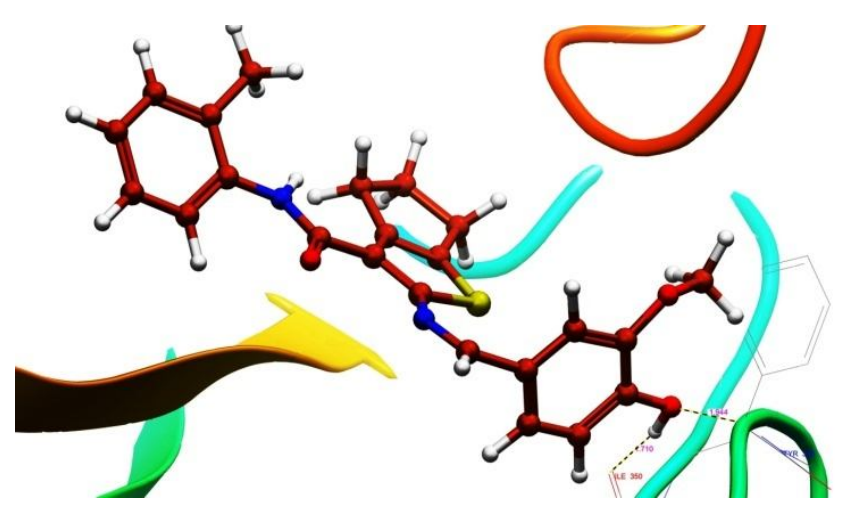

Figure 2: Interaction of compound $2 b$ with the receptor. Docking score of compound $2 b$ was -7.01 interacting with the protein residues Ile 350 and Tyr 348

Flexible docking of all the synthesized compounds was carried out at the active site of GABA-AT. The results reveal that eleven compounds are capable of binding to the active site of the receptor amino acid, Tyr 348; this predicts favorable interaction with protein residues that exhibit hydrophobic and hydrophilic interactions. Protein ligand interaction results indicate that protein residue, such as TYR 348, is the common amino acid that interacted with most of the ligands and other residues such as GLY 104, LEU 103, THR 132, PRO 344, and ILE 350, and that they also participate in the formation of hydrogen bonds. Compounds $2 \mathrm{~b}, 2 \mathrm{e}, 2 \mathrm{f}, 2 \mathrm{o}$ and $2 q$ with 4-hydroxy-3-methoxy substitution showed the highest docked score of 7.01, and were more tightly bound to the active site of GABA-AT than other compounds. The phenyl ring and carbonyl moiety of amide in compound $2 \mathrm{~b}$ displayed good interactions at the active site. The best ligand protein interactions are shown in Figure 2. These observations provide a good basis for the estimation of the inhibitor activity of these compounds. Compounds 2c, $2 \mathrm{~g}, 2 \mathrm{~m}, 2 \mathrm{n}$ and 2s with 4-methylbenzylideneamino , 4isopropylbenzylideneamino, furan-2-ylmethyleneamino, 1H-indol-3-yl-methyleneamino, 4oxopentan-2-ylideneamino at 2-amino-N-o-tolyl- 
5,6-dihydro-4H-Cyclopenta[b]thiophene-3-carboxamide did not demonstrate any hydrogen bond interactions may be responsible for the low docking score.

\section{2-cyano-N-o-tolylacetamide(1):}

Yield (\%):75 , Colour: light pinkish crystals, Mol.wt:174, $\mathrm{R}_{\mathrm{f}}$ value: $0.77, \mathrm{Mp}\left({ }^{0} \mathrm{C}\right)$ : 143-144; IR ( $\left.\mathrm{KBr}, \mathrm{Cm}^{-1}\right): 3268(\mathrm{NH}), 3052(\mathrm{Ar}-\mathrm{H})$,2260(C-N), 1663(C=O); ${ }^{1} \mathrm{H} \quad \mathrm{NMR}\left(\mathrm{CDCl}_{3}, \quad \delta \quad \mathrm{ppm}\right)$ : 2.32(,3H, $\left.\mathrm{CH}_{3}\right), 4.01\left(\mathrm{~d}, 2 \mathrm{H}, \mathrm{CH}_{2}\right), 7.14(\mathrm{~d}, 2 \mathrm{H}, \mathrm{Ar}-\mathrm{H})$, 7.24(d,2H,Ar-CH);Mass (m/z):175[M] ${ }^{+}$. Anal. Calcd.for $\quad \mathrm{C}_{10} \mathrm{H}_{10} \mathrm{~N}_{2} \mathrm{O}$ : $\mathrm{C}(68.95) ; \quad \mathrm{H}(5.79)$; $\mathrm{N}(16.08)$. Found:C(68.85); $\mathrm{H}(5.68) ; \mathrm{N}(15.89)$.

\section{2-amino-N-o-tolyl-5,6-dihydro-4H-} cyclopenta[b]thiophene-3-carboxamide(2):

Yield(\%): 81, Colour: reddish brown crystals Mol.wt: 272, $\mathrm{R}_{\mathrm{f}}$ value: $0.47, \mathrm{Mp}\left({ }^{0} \mathrm{C}\right)$ : 148-150; IR $\left(\mathrm{KBr}, \mathrm{Cm}^{-1}\right): 3429\left(\mathrm{NH}_{2}\right), 3313(\mathrm{NH}), 3021(\mathrm{Ar}-$ $\mathrm{H}), 1628(\mathrm{C}=\mathrm{O}) ;{ }^{1} \mathrm{H}$ NMR $\left(\mathrm{CDCl}_{3}, \delta \mathrm{ppm}\right): 2.30$ ( , $\left.3 \mathrm{H}, \quad \mathrm{CH}_{3}\right), \quad 2.4\left(\mathrm{~m}, 2 \mathrm{H}, \mathrm{CH}_{2}\right), \quad 2.7\left(\mathrm{t}, 2 \mathrm{H}, \mathrm{CH}_{2}\right)$, 6.2(s,2 $\left.\mathrm{H}_{1} \mathrm{NH}_{2}\right), \quad$ 7.03-7.08(t,3H,Ar-H), 7.187.29(m,3H,Ar-H), 7.96-7.99(d,1H,NH);Mass: (m/z):273[M] ${ }^{+}$. Anal.Calcd. for $\mathrm{C}_{15} \mathrm{H}_{16} \mathrm{~N}_{2} \mathrm{OS}$ : C (66.15); $\quad \mathrm{H}$ (5.92); $\mathrm{N}(10.29)$. Found: $\mathrm{C}(66.09)$; $\mathrm{H}(5.90) ; \mathrm{N}(10.32)$.

2-(bezylideneamino)-N-o-tolyl-5,6-dihydro-4Hcyclopenta[b]thiophene-3-carboxamide (2a):

Yield (\%): 57, colour: light greenish crystals, Mol.wt:360, $\mathrm{R}_{\mathrm{f}}$ value: $0.44, \mathrm{Mp}\left({ }^{0} \mathrm{C}\right)$ : 109-110; IR $\left(\mathrm{KBr}, \quad \mathrm{Cm}^{-1}\right)$ : $3365(\mathrm{NH}), 3065(\mathrm{Ar}-\mathrm{H})$, $1569(\mathrm{~N}=\mathrm{CH}), \quad 1668(\mathrm{C}=\mathrm{O}) ;{ }^{1} \mathrm{H} \quad \mathrm{NMR}\left(\mathrm{CDCl}_{3}\right.$, ঠppm): $\quad 2.27\left(\mathrm{~s}, 3 \mathrm{H}, \mathrm{CH}_{3}\right), \quad 2.36-2.45\left(\mathrm{t}, 2 \mathrm{H}, \mathrm{CH}_{2}\right)$, 2.89-2.93(t, $\left.2 \mathrm{H}, \mathrm{CH}_{2}\right), \quad 3.12-3.17\left(\mathrm{t}, 2 \mathrm{H}, \mathrm{CH}_{2}\right), \quad 7.07-$ 7.12(t,1H,Ar-H), 7.80-7.82(d $2 \mathrm{H}, \mathrm{Ar}-\mathrm{H})$ 7.93(d,1H, $\quad$ Ar-H), $8.54(\mathrm{~s}, \mathrm{~N}=\mathrm{CH})$, 10.41(s,1H,NH);Mass(m/z):361[M] ${ }^{+}$.Anal.Calcd.fo $r \quad \mathrm{C}_{22} \mathrm{H}_{20} \mathrm{~N}_{2} \mathrm{OS}: \mathrm{C}$ (73.30); $\mathrm{H} \quad$ (5.59); $\quad \mathrm{N}$ (7.77).Found: C(73.12); H (5.49); N (7.78).

\section{2-(4-hydrox-3-methoxybenzylideneamino)- $\mathrm{N}$ - o-tolyl-5,6-dihydro-4H- \\ cyclopenta[b]thiophene-3-carboxamide(2b):}

Yield (\%): 68\%, colour: dark brown crystals, Mol.wt:406, $\mathrm{R}_{\mathrm{f}}$ value: 0.68, $\mathrm{Mp}\left({ }^{0} \mathrm{C}\right)$ : 190-191; IR $\left(\mathrm{KBr}, \mathrm{Cm}^{-1}\right): 3450(\mathrm{C}-\mathrm{OH}), 3264(\mathrm{NH}), 2947(\mathrm{Ar}-\mathrm{H})$, $1650(\mathrm{C}=\mathrm{O}), \quad 1570(\mathrm{~N}=\mathrm{CH}), \quad 1257\left(\mathrm{Ar}-\mathrm{OCH}_{3}\right) ;{ }^{1} \mathrm{H}$ NMR $\left(\mathrm{CDCl}_{3}, \delta \mathrm{ppm}\right): 2.28\left(\mathrm{~s}, 3 \mathrm{H}, \mathrm{CH}_{3}\right), 2.38-$ $2.4\left(\mathrm{t}, 2 \mathrm{H}, \mathrm{CH}_{2}\right), \quad 2.82-2.92\left(\mathrm{~m}, 3 \mathrm{H}, \mathrm{OCH}_{3}\right), \quad 3.10-$ $3.15\left(\mathrm{t}, 2 \mathrm{H}, \mathrm{CH}_{2}\right), 3.96(\mathrm{~s}, 1 \mathrm{H}, \mathrm{OH}), 3.79\left(\mathrm{~s}, 1 \mathrm{H}, \mathrm{CH}_{2}\right)$, 7.07-7.10(t,1H,Ar-H), 7.18-7.22(m,2H,Ar-H), 7.34-7.36(t,2H,Ar-H), 7.41-7.43(m,1H,Ar-H), 7.86-7.89(d,1H,Ar-H), $\quad$ 8.42(s, $1 \mathrm{H}, \mathrm{N}=\mathrm{CH})$, 10.45(s,1H,NH); Mass (m/z):407[M] ${ }^{+}$. Anal. Calcd.for $\quad \mathrm{C}_{23} \mathrm{H}_{22} \mathrm{~N}_{2} \mathrm{O}_{3} \mathrm{~S}$ : $\mathrm{C}(67.96) ; \quad \mathrm{H}(5.46)$; $\mathrm{N}(6.89)$. Found :C(67.90); $\mathrm{H}(5.43) ; \mathrm{N}(6.86)$.
2-(4-methylbenzylideneamino)-N-o-tolyl-5,6dihydro-4H-cyclopenta[b]thiophene-3carboxamide (2c):

Yield (\%):54, colour: light brownish crystals, Mol.wt:406, $\mathrm{R}_{\mathrm{f}}$ value: $0.70, \mathrm{Mp}\left({ }^{0} \mathrm{C}\right)$ : 130-132; IR $\left(\mathrm{KBr}, \mathrm{Cm}^{-1}\right)$ : 3240(NH), 3077(Ar-H), 2925(CH of $\left.\mathrm{CH}_{3}\right), \quad 1671(\mathrm{C}=\mathrm{O}$ str $), 1584(\mathrm{~N}=\mathrm{CH}) ;{ }^{1} \mathrm{H}$ NMR ( $\left.\mathrm{CDCl}_{3}, \delta \mathrm{ppm}\right): 2.27\left(\mathrm{~m}, 3 \mathrm{H}, \mathrm{CH}_{3}\right), 2.42-2.44(\mathrm{~d}$, $\left.4 \mathrm{H}, \quad \mathrm{CH}_{2}\right), \quad 2.55-2.62\left(\mathrm{~d}, 4 \mathrm{H}, \mathrm{CH}_{2}\right)$, 2.842.97(m,3H, $\left.\mathrm{CH}_{3}\right)$, 7.07-7.23(m,4H,Ar-H), 7.287.34(d,1H,Ar-H), 7.70-7.72(d,1H,Ar-H), 7.767.79(d,1H,Ar-H), 7.88-7.93(d,1H,Ar-H), 8.51 $(\mathrm{s}, 1 \mathrm{H}, \mathrm{N}=\mathrm{CH}), \quad 10.44(\mathrm{~S}, 1 \mathrm{H}, \mathrm{NH}) ; \quad$ Mass $(\mathrm{m} / \mathrm{z})$ : 375[M] ${ }^{+}$. Anal.Calcd. for $\mathrm{C}_{23} \mathrm{H}_{22} \mathrm{~N}_{2} \mathrm{OS}$ : C(73.76); $\mathrm{H}(5.92) ; \quad \mathrm{N}(7.48)$.Found $: \mathrm{C}(73.69) ; \quad \mathrm{H}(5.88)$; $\mathrm{N}(7.45)$.

\section{2-(2-nitrobenzylideneamino)-N-o-tolyl-5,6- dihydro-4H-cyclopenta[b]thiophene-3- carboxamide (2d):}

Yield (\%):66, colour: light brownish crystals, Mol.wt:405, $\quad \mathrm{R}_{\mathrm{f}}$ value: $0.75, \operatorname{Mp}\left({ }^{0} \mathrm{C}\right)$ : 128-130; IR $\left(\mathrm{KBr}, \quad \mathrm{Cm}^{-1}\right): \quad 3285(\mathrm{NH}), \quad 3059(\mathrm{Ar}-\mathrm{H})$, 1664(C=O), 1588(N=CH), 1340 $\left(\mathrm{NO}_{2}\right){ }^{1}{ }^{1} \mathrm{H}$ NMR ( $\mathrm{CDCl}_{3}$, $\left.\quad \delta \quad \mathrm{ppm}\right): \quad 2.12\left(\mathrm{~s}, 3 \mathrm{H}, \mathrm{CH}_{3}\right)$, 2.372.47(m, $\left.2 \mathrm{H}, \mathrm{CH}_{2}\right), \quad 2.90-2.95\left(\mathrm{t}, 2 \mathrm{H}, \mathrm{CH}_{2}\right), \quad 3.13-$ 3.18(t,2H, $\left.\mathrm{CH}_{2}\right), \quad$ 7.16-7.29(m,,2H,Ar-H), 7.727.82(m,3H,Ar-H), 7.96-7.97(d,2H,Ar-H), 8.068.18(s,1H,Ar-H), 9.02(s,1H,N=CH), 10.23(S,1H, $\mathrm{NH})$;Mass (m/z):406[M] ${ }^{+}$Anal.Calcd. for $\mathrm{C}_{22} \mathrm{H}_{19} \mathrm{~N}_{3} \mathrm{O}_{3} \mathrm{~S}: \mathrm{C}(65.17) ; \mathrm{H}(4.72) ; \mathrm{N}(10.36)$. Found $\mathrm{C}(65.20) ; \mathrm{H}(4.69) ; \mathrm{N}(10.32)$.

\section{2-(3-nitrobenzylideneamino)-N-o-tolyl-5,6- dihydro-4H-cyclopenta[b]thiophene-3- carboxamide (2e):}

Yield (\%): 55, colour: dark brown crystals, Mol.wt:405, $\mathrm{R}_{\mathrm{f}}$ value: $0.56, \mathrm{Mp}\left({ }^{0} \mathrm{C}\right)$ : 140-142; IR $\left(\mathrm{KBr}, \quad \mathrm{Cm}^{-1}\right)$ : 3250(NH), 3021(Ar-H ), $1656(\mathrm{C}=\mathrm{O}), 1588(\mathrm{~N}=\mathrm{CH}), 1340\left(\mathrm{NO}_{2}\right) ;{ }^{1} \mathrm{H} \quad \mathrm{NMR}$ $\left(\mathrm{CDCl}_{3}, \quad \delta \quad \mathrm{ppm}\right)$. 1.3(s,1H, $\left.\mathrm{CH}_{3}\right)$, 2.372.5(d, $\left., 2 \mathrm{H}, \mathrm{CH}_{2}\right), \quad 2.7\left(\mathrm{~d}, 2 \mathrm{H}, \mathrm{CH}_{2}\right), \quad 4.2\left(\mathrm{q}, 2 \mathrm{H}, \mathrm{CH}_{2}\right)$, 7.06-7.09(m,2H,Ar-H), 7.64-7.68(t,3H,Ar-H), 7.80-(d,2H,Ar-H), 8.01(s,1H,Ar-H-), 9.2(s,1H, $\mathrm{N}=\mathrm{CH}), \quad 10.12(\mathrm{~s}, 1 \mathrm{H}, \mathrm{NH})$;Mass $(\mathrm{m} / \mathrm{z}): 406[\mathrm{M}]^{+}$. Anal.Calcd.for $\mathrm{C}_{22} \mathrm{H}_{19} \mathrm{~N}_{3} \mathrm{O}_{3} \mathrm{~S}: \mathrm{C}(65.17) ; \mathrm{H}(4.72)$; $\mathrm{N}(10.36)$. Found: $\mathrm{C}(65.20) ; \mathrm{H}(4.69) ; \mathrm{N}(10.32)$.

\section{2-(4-nitrobenzylideneamino)-N-o-tolyl-5,6- dihydro-4H-cyclopenta[b]thiophene-3- carboxamide (2f):}

Yield (\%):59, colour: light brown crystals, Mol.wt:405, $\mathrm{R}_{\mathrm{f}}$ value:0.62 $\mathrm{Mp}\left({ }^{\circ} \mathrm{C}\right)$ : 189-190; IR $\left(\mathrm{KBr}, \mathrm{Cm}^{-1}\right): 3299(\mathrm{NH}), 3122(\mathrm{Ar}-\mathrm{H}), 1631(\mathrm{C}=\mathrm{O})$, $1345\left(\mathrm{NO}_{2}\right) ;{ }^{1} \mathrm{H} \quad \mathrm{NMR} \quad\left(\mathrm{CDCl}_{3}, \delta \mathrm{ppm}\right)$, 1.6 $\left(\mathrm{s}, 3 \mathrm{H}, \mathrm{CH}_{3}\right), \quad 2.4\left(\mathrm{~s}, 2 \mathrm{H}, \mathrm{CH}_{2}\right), \quad 2.50\left(\mathrm{~s}, 1 \mathrm{H}, \mathrm{CH}_{2}\right)$, 4.1 $\left(\mathrm{q}, 2 \mathrm{H}, \mathrm{CH}_{2}\right), \quad 6.9(\mathrm{~s}, 1 \mathrm{H}, \mathrm{Ar}-\mathrm{H}), \quad$ 7.1 $(\mathrm{t}, 3 \mathrm{H}, \mathrm{Ar}-\mathrm{H})$, 7.2(s,3H,Ar-H), 7.4(d,1H,Ar-H), 8.6(s,1H,N=CH), 11.0(s,1H,NH);Mass (m/z):406[M] ${ }^{+}$. Anal.Calcd. 
for $\mathrm{C}_{22} \mathrm{H}_{19} \mathrm{~N}_{3} \mathrm{O}_{3} \mathrm{~S}: \mathrm{C}(65.17) ; \quad \mathrm{H}(4.72) ; \quad \mathrm{N}(10.36)$. Found: $\mathrm{C}(65.20) ; \mathrm{H}(4.69) ; \mathrm{N}(10.32)$.

\section{2-(4-isopropylbenzylideneamino)-N-o-tolyl- 5,6-dihydro-4H-cyclopenta[b]thiophene-3- carboxamide $(\mathbf{2 g})$ :}

Yield (\%):59, colour: dark brown crystals, Mol.wt:402, $\mathrm{R}_{\mathrm{f}}$ value: $0.50 \mathrm{Mp}\left({ }^{0} \mathrm{C}\right)$ : 105-106; IR $\left(\mathrm{KBr}, \mathrm{Cm}^{-1}\right):$ 3295(NH), 3040(Ar-H), 2971(Ali$\mathrm{CH}), 1645(\mathrm{C}=\mathrm{O}), 1583(\mathrm{~N}=\mathrm{CH}), 1366\left(\left(\mathrm{CH}_{3}\right)_{2}\right) ;{ }^{1} \mathrm{H}$ NMR ( $\left.\mathrm{CDCl}_{3}, \delta \mathrm{ppm}\right): 1.26-1.29\left(\mathrm{~d}, 6 \mathrm{H},\left(\mathrm{CH}_{3}\right)_{2}\right)$, $2.29\left(\mathrm{~s}, 1 \mathrm{H}, \mathrm{CH}_{3}\right), \quad 2.37-2.45\left(\mathrm{t}, 2 \mathrm{H}, \mathrm{CH}_{2}\right), \quad 2.84-$ 3.01(m,1H, CH), 3.12-3.16(t,2H, $\left.\mathrm{CH}_{2}\right)$, $\quad$ 7.227.40(m,5H,Ar-H), 7.73-7.75(d,2H,Ar-H), 7.88$7.91 \quad(\mathrm{~d}, 1 \mathrm{H}, \mathrm{Ar}-\mathrm{H}), 8.50(\mathrm{~s}, 1 \mathrm{H}, \mathrm{N}=\mathrm{CH})$, 10.47(s,1H,NH); Mass (m/z):403[M] ${ }^{+}$. Anal.Calcd. for $\quad \mathrm{C}_{25} \mathrm{H}_{26} \mathrm{~N}_{2} \mathrm{OS}: \mathrm{C}(74.59) ; \quad \mathrm{H}(6.51)$; $\mathrm{N}(6.96)$.Found: $\mathrm{C}(74.52) ; \mathrm{H}(6.52) ; \mathrm{N}(6.98)$.

\section{2-(4-fluorobenzylideneamino)-N-o-tolyl-5,6- dihydro-4H-cyclopenta[b]thiophene-3- carboxamide (2h):}

Yield (\%): 80, colour: lemon yellow crystals, Mol.wt:378, $\mathrm{R}_{\mathrm{f}}$ value: 0.76, $\mathrm{Mp}\left({ }^{0} \mathrm{C}\right): 165-166$ ; IR $\left(\mathrm{KBr}, \mathrm{Cm}^{-1}\right):$ 3292(NH), 3070(Ar1666(C=O), $1587(\mathrm{~N}=\mathrm{CH}), 1229(\mathrm{C}-\mathrm{F}) ;{ }^{1} \mathrm{H}$ NMR ( $\left.\mathrm{CDCl}_{3}, \quad \delta \quad \mathrm{ppm}\right): 2.25\left(\mathrm{~S}, 3 \mathrm{H}, \mathrm{CH}_{3}\right), 2.36-$ 2.45(m,2H, $\left.\mathrm{CH}_{2}\right), 2.88-2.93\left(\mathrm{t}, 2 \mathrm{H}, \mathrm{CH}_{2}\right), 3.12-$

3.16(t,2H, $\left.\mathrm{CH}_{2}\right)$, 7.23(m,5H,Ar-H), 7.797.84(t,2H,Ar-H), 7.90-7.93(d,1H,Ar-H),8.5(s,1H, $\mathrm{N}=\mathrm{CH}), 10.31(\mathrm{~s}, 1 \mathrm{H}, \mathrm{NH}) ; \quad(\mathrm{m} / \mathrm{z}): 379[\mathrm{M}]^{+}$. Anal.Cal cd.for $\quad \mathrm{C}_{22} \mathrm{H}_{19} \mathrm{FN}_{2} \mathrm{OS}$ : $\mathrm{C}(69.82) ; \quad \mathrm{H}(5.06)$; $\mathrm{N}(7.40)$ :Found: C(69.80); H(5.08); N(7.38).

\section{2-(4-(dimethylamino)bezylideneamino)-N-o-} tolyl-5,6-dihydro-4H-cyclopenta[b]thiophene3-carboxamide (2i):

Yield (\%):62, colour: dark reddish crystals, Mol.wt:403, $\mathrm{R}_{\mathrm{f}}$ value:0.50, $\mathrm{Mp}\left({ }^{0} \mathrm{C}\right)$ : 160-161; IR $\left(\mathrm{KBr}, \mathrm{Cm}^{-1}\right)$ : 3219(NH), 3024(Ar-H), 2906(CH Of $\left.\mathrm{CH}_{3}\right), \quad 1660(\mathrm{C}=\mathrm{O}), 1584(\mathrm{~N}=\mathrm{CH}) ;{ }^{1} \mathrm{H}$ NMR ( $\left.\mathrm{CDCl}_{3}, \quad \delta \mathrm{ppm}\right): \quad 2.29\left(\mathrm{~s}, \quad 3 \mathrm{H}, \mathrm{CH}_{3}\right), \quad 2.36-$ 2.40(t,2 $\left.\mathrm{H}, \mathrm{CH}_{2}\right), \quad 2.86-2.91\left(\mathrm{t}, 4 \mathrm{H}, \mathrm{CH}_{2}\right), \quad 3.10-$ $3.15\left(\mathrm{~s}, 6 \mathrm{H},\left(\mathrm{CH}_{3}\right)_{2}\right), \quad 6.92-6.95(\mathrm{~d}, 2 \mathrm{H}, \mathrm{Ar}-\mathrm{H}), \quad 7.06-$ 7.11(t,1H,Ar-H), 7.20-7.23(t,2H,Ar-H), 7.737.71(d,2H,Ar-H), 7.88-7.91(d,1H,Ar-H), 8.4( $\mathrm{S}, 1 \mathrm{H}, \mathrm{N}=\mathrm{CH}), \quad$ 10.51 $(\mathrm{s}, 1 \mathrm{H}, \mathrm{NH}) ; \quad$ Mass $(\mathrm{m} / \mathrm{z}): 404[\mathrm{M}]^{+}$. Anal.Calcd.for $\mathrm{C}_{24} \mathrm{C}_{25} \mathrm{~N}_{3} \mathrm{OS}$ : $\mathrm{C}(71.43) ; \mathrm{H}(6.24) ; \mathrm{N}(10.41)$. Found:C(71.35); $\mathrm{H}(6.14) ; \mathrm{N}(10.11)$.

\section{2-(2,4-dichlorobenzylideneamino)-N-o-tolyl- 5,6-dihydro-4H-cyclopenta[b]thiophene-3- carboxamide (2j):}

Yield (\%):57, colour: light brown crystals, Mol.wt:428, $\mathrm{R}_{\mathrm{f}}$ value:0.77, $\mathrm{Mp}\left({ }^{0} \mathrm{C}\right): 180-182$. IR $\left(\mathrm{KBr}, \mathrm{Cm}^{-1}\right):$ 3178(NH), 3036(Ar-H), 1661(C=O ), 1575(N=CH); $\quad{ }^{1} \mathrm{H} \quad \mathrm{NMR} \quad\left(\mathrm{CDCl}_{3}, \quad \delta\right.$ ppm):2.18(s, $\left.1 \mathrm{H}, \mathrm{CH}_{3}\right), \quad 2.39-2.49\left(\mathrm{~m}, 2 \mathrm{H}, \mathrm{CH}_{2}\right)$, 2.83-2.88(t,2H, $\left.\mathrm{CH}_{2}\right)$, 2.92-2.97(t,2H, $\left.\mathrm{CH}_{2}\right), \quad 7.30-$
7.39(m,4H,Ar-H),7.48-7.49(S,1H,Ar-H),7.86-

7.96(m,2H,ArH),9.54(s,1H,N=CH),10.41(s,1H,N $\mathrm{H}) ; \operatorname{Mass}(\mathrm{m} / \mathrm{z}): 429 \mathrm{M}^{+}, 431[\mathrm{M}]^{+2}$. Anal.Calcd. for $\mathrm{C}_{22} \mathrm{H}_{18} \mathrm{Cl}_{2} \mathrm{~N}_{2} \mathrm{OS}$ : $\mathrm{C}(61.54) ; \mathrm{H}(4.23)$;

$\mathrm{N}(6.52)$.Found: $\mathrm{C}(61.49) ; \mathrm{H}(4.18) ; \mathrm{N}(6.56)$.

\section{2-(2-chlorobenzylideneamino)-N-o-tolyl-5,6-} dihydro-4H-cyclopenta[b]thiophene-3carboxamide (2k):

Yield (\%):70, colour: dark brown crystals, Mol.wt:394, $\mathrm{R}_{\mathrm{f}}$ value: 0.73, $\mathrm{Mp}\left({ }^{0} \mathrm{C}\right)$ : 208-210; IR $\left(\mathrm{KBr}, \mathrm{Cm}^{-1}\right)$ : 3290(NH), 3070(Ar-H), 1671(C=O), 1553(N=CH); ${ }^{1} \mathrm{H}$ NMR ( $\left.\mathrm{CDCl}_{3}, \delta \mathrm{ppm}\right)$ : $2.21\left(\mathrm{~s}, 3 \mathrm{H}, \mathrm{CH}_{3}\right), \quad 2.36-2.46\left(\mathrm{~m}, 2 \mathrm{H}, \mathrm{CH}_{2}\right)$, 2.892.94(t,2 $\left.\mathrm{H}, \mathrm{CH}_{2}\right), \quad 3.13-3.17\left(\mathrm{t}, 3 \mathrm{H}, \mathrm{CH}_{2}\right), \quad$ 7.067.11(t,1H,Ar-H), 7.18-7.42(m,3H,Ar-H), 7.447.48(s,1H,Ar-H), 7.91-7.99(m,2H,Ar-H), 8.95(s,1H,Ar-H), 10.32(S,1H,N=CH), 10.49(s,1H,NH); Mass(m/z):395[M] ${ }^{+}, \quad 397[\mathrm{M}]^{+2}$. Anal.Calcd. for $\mathrm{C}_{22} \mathrm{H}_{19} \mathrm{CIN}_{2} \mathrm{OS}: \mathrm{C}(66.91) ; \mathrm{H}(4.85) ; \mathrm{N}(7.09)$.Found: $\mathrm{C}(66.85) ; \mathrm{H}(4.88) ; \mathrm{N}(7.14)$.

2-(2,4-dimethoxybenzylideneamino)-N-o-tolyl5,6-dihydro-4H-cyclopenta[b]thiophene-3carboxamide (2I):

Yield (\%):55, colour: Apple green crystals, Mol.wt:420, $\mathrm{R}_{\mathrm{f}}$ value:0.59, Mp $\left({ }^{0} \mathrm{C}\right)$ : 142-144; IR $\left(\mathrm{KBr}, \mathrm{Cm}^{-1}\right): 3178(\mathrm{NH}), 3036(\mathrm{Ar}-\mathrm{H}), 1661(\mathrm{C}=\mathrm{O})$, 1575(N=CH), $1269\left(\mathrm{Ar}-\mathrm{OCH}_{3}\right) ;{ }^{1} \mathrm{H} \mathrm{NMR}\left(\mathrm{CDCl}_{3}, \delta\right.$ ppm): $\quad 2.17\left(\mathrm{~s}, 2 \mathrm{H}, \mathrm{CH}_{2}\right), \quad 2.28-2.31\left(\mathrm{~s}, 2 \mathrm{H}, \mathrm{CH}_{3}\right)$, $2.40\left(\mathrm{~s}, 2 \mathrm{H}, \mathrm{CH}_{2}\right), \quad 2.43-2.46\left(\mathrm{~m}, 3 \mathrm{H}, \mathrm{OCH}_{3}\right), 2.83-$ $.96\left(\mathrm{~m}, 3 \mathrm{H}, \mathrm{OCH}_{3}\right), \quad 3.11-3.16\left(\mathrm{t}, 2 \mathrm{H}, \mathrm{CH}_{2}\right), \quad 7.29-$ 7.42(m,6H,Ar-H), 7.85-7.88(s,1H,Ar-H), 8.44(S,1H,N=CH), $\quad$ 9.86(s, $1 \mathrm{H}, \mathrm{NH})$;Mass $(\mathrm{m} / \mathrm{z}): 421[\mathrm{M}]^{+}$. Anal.Calcd. for $\mathrm{C}_{24} \mathrm{H}_{24} \mathrm{~N}_{2} \mathrm{O}_{3} \mathrm{~S}: \mathrm{C}(68.55) ; \mathrm{H}(5.75) ; \mathrm{N}(6.66)$.Found $: \mathrm{C}(68.45) ; \mathrm{H}(5.69) ; \mathrm{N}(6.58)$.

2-(furan-2-ylmethyleneamino)-N-o-tolyl-5,6dihydro-4H-cyclopenta[b]thiophene-3carboxamide $(2 \mathrm{~m})$ :

Yield (\%):71, colour: light green crystals, Mol.wt:350, $\mathrm{R}_{\mathrm{f}}$ value: 0.64, Mp $\left({ }^{0} \mathrm{C}\right)$ : 138-140; IR $\left(\mathrm{KBr}, \quad \mathrm{Cm}^{-1}\right): \quad 3287(\mathrm{NH}), \quad 3057(\mathrm{Ar}-\mathrm{CH} \quad \mathrm{Str})$, 1669(C=O Str), 1587( $\mathrm{N}=\mathrm{CH}-\mathrm{str}) ;{ }^{1} \mathrm{H}$ NMR ( $\mathrm{CDCl}_{3}$, $\left.\delta \mathrm{ppm}\right): 2.35-2.44\left(\mathrm{~m}, 4 \mathrm{H}, \mathrm{CH}_{2}\right), 2.83-$ 2.97(m,3H, $\left.\mathrm{CH}_{3}\right), 3.12-3.17\left(\mathrm{t}, 2 \mathrm{H}, \mathrm{CH}_{2}\right), 6.99(\mathrm{~d}, 1 \mathrm{H}$, Ar-H), 7.05(t,1H,Ar-H), 7.20(t,2H,Ar-H), 7.33(s,1H,Ar-H), 7.62(s,1H,Ar-H), 8.05(d,1H,Ar$\mathrm{H}), \quad 8.25(\mathrm{~s}, 1 \mathrm{H}, \mathrm{N}=\mathrm{CH}), \quad 10.64(\mathrm{~s}, 1 \mathrm{H}, \mathrm{NH})$; Mass (m/z):351[M] ${ }^{+}$. Anal.Calcd. for $\mathrm{C}_{20} \mathrm{H}_{18} \mathrm{~N}_{2} \mathrm{O}_{2} \mathrm{~S}$ : $\mathrm{C}(68.55) ; \mathrm{H}(5.18) ; \mathrm{N}(7.99)$.Found:C(69.12); $\mathrm{H}(5$. $05) ; \mathrm{N}(7.86)$.

2-(1H-indol-3-yl)methyleneamino)-N-o-tolyl5,6-dihydro-4H-cyclopenta[b]thiophene-3carboxamide $(2 n)$ : 
Yield (\%):69, colour: dark yellow crystals, Mol.wt:399, $\mathrm{R}_{\mathrm{f}}$ value: $0.54, \mathrm{Mp}\left({ }^{\circ} \mathrm{C}\right)$ : $180-182$; IR $(\mathrm{KBr}$,

$\left(m^{-1}\right)$ :

$3430,3223(\mathrm{NH}), 1655(\mathrm{C}=\mathrm{O}), 1612(\mathrm{C}=\mathrm{O}$

indole), $1588(\mathrm{~N}=\mathrm{CH}) ;{ }^{1} \mathrm{H}$ NMR ( DMSO, $\delta \mathrm{ppm}$ ): $2.05\left(\mathrm{~s}, 3 \mathrm{H}, \mathrm{CH}_{3}\right), \quad 2.27-2.37\left(\mathrm{~m}, 2 \mathrm{H}, \mathrm{CH}_{2}\right), \quad$ 2.83$2.88\left(\mathrm{~s}, 2 \mathrm{H}, \mathrm{CH}_{2}\right), \quad 2.95-2.97\left(\mathrm{~s}, 2 \mathrm{H}, \mathrm{CH}_{2}\right), \quad 6.80-$ 6.85(t,1H,Ar-H), $\quad 7.09-7.26(\mathrm{~m}, 4 \mathrm{H}, \mathrm{Ar}-\mathrm{H}), \quad$ 7.497.52(d,1H,Ar-H), 7.67-7.70(d,1H,Ar-H), 7.917.94(d,1H, Ar-H),8.26-8.27(s,1H,Ar-H),8.80(s,1H, $\mathrm{N}=\mathrm{CH}), 10.61(\mathrm{~s}, 1 \mathrm{H}, \mathrm{NH}), \quad 12.15(\mathrm{~s}, 1 \mathrm{H}, \mathrm{NH}$ indole); Mass $\quad(\mathrm{m} / \mathrm{z}): 400[\mathrm{M}]^{+}$. Anal.Calcd. for $\mathrm{C}_{24} \mathrm{H}_{21} \mathrm{~N}_{3} \mathrm{OS}: \mathrm{C}(72.15) ; \mathrm{H}(5.30) ; \mathrm{N}(10.52)$. Found: $\mathrm{C}(72.20) ; \mathrm{H}(5.18) ; \mathrm{N}(10.32)$.

2-(2-oxoindolin-3-ylideneamino)-N-o-tolyl-5,6dihydro-4H-cyclopenta[b]thiophene-3carboxamide (20):

Yield (\%):32, colour: dark reddish crystals, Mol.wt:401, $\mathrm{R}_{\mathrm{f}}$ value:0.83, $\mathrm{Mp}\left({ }^{0} \mathrm{C}\right)$ : 149-150; IR $\left(\mathrm{KBr}, \mathrm{Cm}^{-1}\right)$ : 3296(NH), 3042(Ar-H), 1666(C=O), 1606 $(\mathrm{C}=\mathrm{O}, \quad$ isatin $), \quad 1589(\mathrm{~N}=\mathrm{CH}) ;{ }^{1} \mathrm{H} \quad \mathrm{NMR} \quad($ DMSO, $\delta$ ppm): $2.30\left(\mathrm{~s}, 3 \mathrm{H}, \mathrm{CH}_{3}\right), \quad 2.45-$ $2.52\left(\mathrm{t}, 2 \mathrm{H}, \mathrm{CH}_{2}\right), 2.79\left(\mathrm{~s}, 2 \mathrm{H}, \mathrm{CH}_{2}\right), 2.87-2.95(\mathrm{~s}, 2 \mathrm{H}$, $\left.\mathrm{CH}_{2}\right), \quad 7.15-7.22(\mathrm{~m}, 7 \mathrm{H}, \mathrm{Ar}-\mathrm{H}), \quad 7.43(\mathrm{~s}, 1 \mathrm{H}, \mathrm{Ar}-\mathrm{CH})$, 8.00(s, $1 \mathrm{H}, \mathrm{NH}), \quad 11.75(\mathrm{~s}, 1 \mathrm{H}$,lactam); Mass $(\mathrm{m} / \mathrm{z})$ : 402[M] ${ }^{+}$. Anal.Calcd.for ${ }_{24} \mathrm{H}_{21} \mathrm{~N}_{3} \mathrm{O}_{2} \mathrm{~S}$ : C(69.37); $\mathrm{H}(5.09)$; $\mathrm{N}(10.11)$.Found: $\mathrm{C}(68.37) ; \mathrm{H}(4.10) ; \mathrm{N}$ (10.15).

\section{2-(cyclohexylideneamino)-N- o-tolyl-5,6- dihydro-4H-cyclopenta[b]thiophene-3- carboxamide (2p):}

Yield (\%):58, colour: dark brown amorphous powder, Mol.wt:352, $\mathrm{R}_{\mathrm{f}}$ value:0.65 Mp $\left({ }^{0} \mathrm{C}\right)$ : 102103; IR $\left(\mathrm{KBr}, \mathrm{Cm}^{-1}\right): 3298(\mathrm{NH}), 3056(\mathrm{Ar}-\mathrm{H})$, 2930(Alip-CH), 1630(C=O), 1573(C=N ); ${ }^{1} \mathrm{H}$ NMR ( $\left.\mathrm{CDCl}_{3}, \quad \delta \mathrm{ppm}\right): \quad 2.15\left(\mathrm{~s}, 3 \mathrm{H}, \mathrm{CH}_{3}\right), \quad 2.37-$ $2.30\left(\mathrm{~m}, 6 \mathrm{H}, \mathrm{CH}_{2}\right), \quad 2.43-2.57\left(\mathrm{~m}, 2 \mathrm{H}, \mathrm{CH}_{2}\right), \quad 2.83-$ $3.08\left(\mathrm{~m}, 6 \mathrm{H}, \mathrm{CH}_{2}\right), \quad 3.41\left(\mathrm{~S}, 2 \mathrm{H}, \mathrm{CH}_{2}\right), 7 . \quad 19-$ $7.23(\mathrm{~m}, 4 \mathrm{H}, \quad \operatorname{Ar}-\mathrm{H}), \quad 8.02(\mathrm{~N}=\mathrm{CH}), \quad 11.79(\mathrm{~s}, 1 \mathrm{H}$, $\mathrm{NH})$;Mass $(\mathrm{m} / \mathrm{z}): 351[\mathrm{M}]$. Anal.Calcd. for $\mathrm{C}_{22} \mathrm{H}_{26} \mathrm{~N}_{2} \mathrm{OS}$ : $\mathrm{C}(72.09) ; \mathrm{H}(7.15) ; \mathrm{N}(7.64)$. Found: $\mathrm{C}(73.01) ; \mathrm{H}(7.25) ; \mathrm{N}(7.62)$.

\section{2-(1-methylpyrrolidin-2-ylideneamino)-N- o- tolyl-5,6-dihydro-4H-cyclopenta[b]thiophene- 3-carboxamide (2q):}

Yield (\%):49, colour: dark brown amorphous powder, Mol.wt:353, $\mathrm{R}_{\mathrm{f}}$ value:0.69, Mp $\left({ }^{0} \mathrm{C}\right): 138-$ 139; IR ( $\mathrm{KBr}$ in $\left.\mathrm{Cm}^{-1}\right): 3313(\mathrm{NH}), 2929(\mathrm{Ar}-\mathrm{H})$, 1628(C=O), 1572(C=N),1341(C-N); ${ }^{1} \mathrm{H} \quad$ NMR $\left(\mathrm{CDCl}_{3}, \quad \delta \quad \mathrm{ppm}\right): \quad 1.13 \quad\left(\mathrm{~s}, 3 \mathrm{H}, \mathrm{NCH}_{3}\right), \quad 2.34$ $\left(\mathrm{S}, 3 \mathrm{H}, \mathrm{CH}_{3}\right), \quad 4.0\left(\mathrm{~d}, 2 \mathrm{H}, \mathrm{CH}_{2}\right), \quad 2.5\left(\mathrm{~d}, 2 \mathrm{H}, \mathrm{CH}_{2}\right)$, $2.8\left(\mathrm{~d}, 2 \mathrm{H}, \mathrm{CH}_{2}\right), \quad 7.96(\mathrm{~s}, 1 \mathrm{H}, \mathrm{NH}), \quad 7.27(\mathrm{~m}, 4 \mathrm{H}, \mathrm{Ar}-$ $\mathrm{CH})$; Mass $(\mathrm{m} / \mathrm{z}): 354[\mathrm{M}]^{+}$. Anal.Calcd. for $\mathrm{C}_{21} \mathrm{H}_{25} \mathrm{~N}_{3} \mathrm{OS}$ : $\mathrm{C}(68.63) ; \mathrm{H}(6.86) ; \mathrm{N}(11.43)$. Found $: \mathrm{C}(68.43) ; \mathrm{H}(6.74) ; \mathrm{N}(11.89)$.
2-(butan-2-ylideneamino)-N-o-tolyl-5,6dihydro-4H-Cyclopenta[b]thiophene-3carboxamide(2r):

Yield (\%):71 colour:Ash coloured crystals, Mol.wt:326, $\mathrm{R}_{\mathrm{f}}$ value:0.60, $\operatorname{Mp}\left({ }^{\circ} \mathrm{C}\right): 115-117$; IR $\left(\mathrm{KBr}\right.$ in $\left.\mathrm{Cm}^{-1}\right): 3314(\mathrm{NH}), 2904(\mathrm{Ar}-\mathrm{H}), 2843(\mathrm{CH}$ str in $\left.\mathrm{CH}_{3}\right), 1628(\mathrm{C}=\mathrm{O} \mathrm{Str}), 1571(\mathrm{C}=\mathrm{N} \mathrm{Str}) ;{ }^{1} \mathrm{H}$ NMR $\left(\mathrm{CDCl}_{3}, \quad \delta \mathrm{ppm}\right): \quad 1.4\left(\mathrm{t}, 6 \mathrm{H}, \mathrm{CH}_{3}\right)$, $2.4\left(\mathrm{~m}, 3 \mathrm{H}, \mathrm{CH}_{3}\right), 2.9\left(\mathrm{~m}, 4 \mathrm{H}, \mathrm{CH}_{2}\right), 4.3(\mathrm{~m}, 4 \mathrm{H}, \mathrm{CH} 2)$, 9.57(s, NH,1H), ,8.44(d,1H,Ar-H), 8.02(d,1H,Ar$\mathrm{H}), \quad 7.7(\mathrm{t}, 1 \mathrm{H}, \mathrm{Ar}-\mathrm{H}), \quad 7.54(\mathrm{t}, 1 \mathrm{H}, \mathrm{Ar}-\mathrm{H}) ; \quad$ Mass $(\mathrm{m} / \mathrm{z}): 327[\mathrm{M}]^{+}$. Anal.Calcd.for $\mathrm{C}_{19} \mathrm{H}_{22} \mathrm{~N}_{2} \mathrm{OS}: \mathrm{C}(69$. 90); $\mathrm{H}(6.79) ; \mathrm{N}(8.58)$. Found: $\mathrm{C}(68.54) ; \mathrm{H}(6.53)$; $\mathrm{N}(8.12)$.

2-(4-oxopentan-2-ylideneamino)-N-o-tolyl-5,6dihydro-4H-cyclopenta[b]thiophene-3carboxamide(2s):

Yield (\%):55, colour:light brown crystals, Mol.wt:354, $R_{\mathrm{f}}$ value:0.71, $\operatorname{Mp}\left({ }^{0} \mathrm{C}\right): 120-122 ; \mathrm{IR}$ $\left(\mathrm{KBr}\right.$ in $\left.\mathrm{Cm}^{-1}\right): 3314(\mathrm{NH}), 3056(\mathrm{Ar}-\mathrm{H}), 2850(\mathrm{CH}-$ Str in $\left.\mathrm{CH}_{3}\right), 1628(\mathrm{C}=\mathrm{O}), 1571(\mathrm{C}=\mathrm{N}) ;{ }^{1} \mathrm{H}$ NMR ( $\left.\mathrm{CDCl}_{3}, \delta \mathrm{ppm}\right): \quad 1.2\left(\mathrm{t}, 6 \mathrm{H}, \mathrm{CH}_{3}\right), \quad 1.6\left(\mathrm{~s}, 3 \mathrm{H}, \mathrm{CH}_{3}\right)$, $2.5\left(\mathrm{t}, 6 \mathrm{H}, \mathrm{CH}_{2}\right), \quad 4.1\left(\mathrm{~m}, 2 \mathrm{H}, \mathrm{CH}_{2}\right), \quad 6.83(\mathrm{~d}, 1 \mathrm{H}, \mathrm{Ar}-$ $\mathrm{H}), 7.0(\mathrm{t}, 1 \mathrm{H}, \mathrm{CH}), 7.12(\mathrm{t}, 1 \mathrm{H}, \mathrm{Ar}-\mathrm{H}), 7.32(\mathrm{~s}, 1 \mathrm{H}, \mathrm{d})$, 10.5(s,1H,NH); Mass (m/z):355[M] ${ }^{+}$. Anal.Calcd. for ${ }_{20} \mathrm{H}_{22} \mathrm{~N}_{2} \mathrm{O}_{2} \mathrm{~S}: \mathrm{C}(67.77) ; \mathrm{H}(6.26) ; \mathrm{N}(7.90)$. Found: $\mathrm{C}(66.15), \mathrm{H}(5.89) ; \mathrm{N}(7.14)$.

\section{2-(1-phenylethylideneamino)-N- o-tolyl-5,6- dihydro-4H-cyclopenta[b]thiophene-3- carboxamide (2t):}

Yield (\%):60, colour:dark brown crystals, Mol.wt:375, $R_{f}$ value: $0.59 \mathrm{Mp}\left({ }^{0} \mathrm{C}\right)$ : $124-125$;IR $\left(\mathrm{KBr}\right.$ in $\mathrm{Cm}^{-1}$ ). 3315(-NH), 2923(Ar-H), 2851(Alip$\left.\mathrm{CH}_{2}\right), 1628(\mathrm{C}=\mathrm{O} \quad \mathrm{Str}), 1571(\mathrm{C}=\mathrm{N} \quad \mathrm{Str}){ }^{1} \mathrm{H} \quad \mathrm{NMR}$ $\left(\mathrm{CDCl}_{3}, \delta \mathrm{ppm}\right) \cdot 1.8\left(\mathrm{~s}, 3 \mathrm{H}, \mathrm{CH}_{3}\right), 2.1\left(\mathrm{~s}, 3 \mathrm{H}, \mathrm{CH}_{3}\right)$, $2.60\left(\mathrm{~m}, 2 \mathrm{H}, \mathrm{CH}_{2}\right), \quad 2.92\left(\mathrm{t}, 2 \mathrm{H}, \mathrm{CH}_{2}\right), \quad 2.4\left(\mathrm{t}, 2 \mathrm{H}, \mathrm{CH}_{2}\right)$, 7.0 (m,5H,Ar-H), 7.32(d,1H,Ar-H), 7.45(s,1H,Ar$\mathrm{H}), \quad 7.65(\mathrm{~m}, 2 \mathrm{H}, \mathrm{Ar}-\mathrm{H}), 8.45(\mathrm{~s}, 1 \mathrm{H}, \mathrm{NH})$; Mass $(\mathrm{m} / \mathrm{z}): 376[\mathrm{M}]^{+} . \quad \mathrm{C}_{23} \mathrm{H}_{22} \mathrm{~N}_{2} \mathrm{OS}: \mathrm{C}(73.76) ; \mathrm{H}(5.92) ;$ $\mathrm{N}(7.48)$. Found $\mathrm{C}(72.87) ; \mathrm{H}(5.46) ; \mathrm{N}(7.12)$.

\section{DISCUSSION}

The compounds synthesized by both microwave and conventional method. 2-Amino-N- o-tolyl-5,6dihydro-4H-cyclopenta[b]thiophene-3-carboxamide was prepared by the reaction of 2-cyano- $\mathrm{N}$ o-tolylacetamide with cyclohexanone, sulphur and ionic liquid. The IR spectrum of 2-cyano-N-otolylacetamide showed the presence of $\mathrm{CN}$ peak at $2260 \mathrm{~cm}^{-1}(\mathrm{C}-\mathrm{N})$, disappearance of $\mathrm{CN}$ peak and formation of $\mathrm{NH}_{2}$ peak at $3429 \mathrm{~cm}^{-1}$, and thus confirmed the formation of 2aminothiophenes when basic ionic liquids are used for synthesis. Ionic liquids are non-volatile, recylable, non-explosive, thermally robust. 2Amino-N-tolyl-5,6-dihydro-4H- cyclopenta(b) 
Table 1: Anticonvulsant activity of 2-(Substituted)-N- o-tolyl-5,6-dihydro-4H-cyclopenta[b]thiophene-3carboxamide on Maximum Electroshock induced convulsions(MES)

\begin{tabular}{|c|c|c|c|c|c|c|}
\hline Group & Treatment & Flexion (s) & Extension (s) & Clonus (s) & Stupor (s) & Recovery (s) \\
\hline I & Control & $6.81 \pm 0.016$ & $12.81 \pm 0.006$ & $13.28 \pm 0.040$ & $6.21 \pm 0.040$ & 189.5 \\
\hline II & Phenytoin & $4.83 \pm 0.001^{* \star *}$ & $0.00 \pm 0.000^{* \star *}$ & $8.31 \pm 0.0166^{\star \star \star}$ & $0.98 \pm 0.016^{\star \star *}$ & 17.3 \\
\hline III & 2 & $6.11 \pm 0.016^{*}$ & $11.8 \pm 0.018^{*}$ & $12.65 \pm 0.004^{*}$ & $5.72 \pm 0.007^{*}$ & 82.1 \\
\hline IV & $2 a$ & $5.18 \pm 0.016^{* * *}$ & $3.83 \pm 0.021^{* \star \star}$ & $10.28 \pm 0.090^{* \star \star}$ & $4.88 \pm 0.007^{* \star *}$ & 75.6 \\
\hline V & $2 b$ & $1.78 \pm 0.116^{* \star \star}$ & $1.55 \pm 0.022^{* \star *}$ & $1.11 \pm 0.030^{* \star \star}$ & $0.97 \pm 0.004^{* * *}$ & 68.3 \\
\hline VI & $2 c$ & $4.21 \pm 0.006^{\star \star \star}$ & $2.18 \pm 0.016^{* * *}$ & $4.40 \pm 0.036^{* * *}$ & $1.83 \pm 0.049^{* * *}$ & 70.3 \\
\hline VII & $2 d$ & $2.71 \pm 0.060^{* \star *}$ & $2.05 \pm 0.022^{* \star \star}$ & $1.93 \pm 0.021^{* \star *}$ & $1.21 \pm 0.001^{* \star *}$ & 69.5 \\
\hline VIII & $2 e$ & $3.25 \pm 0.057^{\star \star \star \star}$ & $2.76 \pm 0.017^{\star \star \star}$ & $2.20 \pm 0.001^{\star \star \star}$ & $1.81 \pm 0.009^{* * *}$ & 78.1 \\
\hline IX & $2 f$ & $2.71 \pm 0.001^{\star \star *}$ & $2.24 \pm 0.006^{* * *}$ & $1.98 \pm 0.002^{* * *}$ & $1.46 \pm 0.021^{* \star *}$ & 72.3 \\
\hline$x$ & $2 g$ & $3.92 \pm 0.002^{* \star *}$ & $3.50 \pm 0.002^{* \star *}$ & $8.75 \pm 0.150$ & $1.31 \pm 0.098^{* * *}$ & 69.5 \\
\hline$X I$ & $2 \mathrm{~h}$ & $3.85 \pm 0.006^{\star \star \star}$ & $3.42 \pm 0.003^{\star * \star}$ & $8.32 \pm 0.076^{\star \star \star}$ & $1.53 \pm 0.111^{\star \star \star}$ & 78.1 \\
\hline XII & $2 \mathrm{i}$ & $5.62 \pm 0.004^{* \star *}$ & $4.81 \pm 0.006^{\star \star \star}$ & $8.02 \pm 0.014^{\star * *}$ & $1.48 \pm 0.065^{\star \star *}$ & 72.3 \\
\hline XIII & $2 \mathrm{j}$ & $4.51 \pm 0.010^{* * *}$ & $3.33 \pm 0.008^{* \star \star}$ & $8.14 \pm 0.014^{\star * \star *}$ & $1.23 \pm 0.084^{* \star *}$ & 77.5 \\
\hline XIV & $2 \mathrm{k}$ & $2.85 \pm 0.009^{\star \star \star}$ & $2.14 \pm 0.001^{\star \star *}$ & $5.80 \pm 0.001^{\star \star *}$ & $1.31 \pm 0.003^{\star \star *}$ & 89.2 \\
\hline$X V$ & 21 & $3.28 \pm 0.030^{* * *}$ & $3.05 \pm 0.034^{* * *}$ & $8.23 \pm 0.156$ & $1.85 \pm 0.194^{* \star *}$ & 87.8 \\
\hline $\mathrm{XVI}$ & $2 m$ & $2.91 \pm 0.003^{* \star *}$ & $2.51 \pm 0.006^{* \star *}$ & $7.63 \pm 0.130^{* \star \star}$ & $0.95 \pm 0.011^{* \star *}$ & 84.4 \\
\hline XVII & $2 n$ & $2.68 \pm 0.003^{* \star \star}$ & $2.50 \pm 0.002^{\star \star \star}$ & $7.63 \pm 0.055^{\star \star \star}$ & $0.96 \pm 0.014^{* * *}$ & 92.0 \\
\hline XVIII & 20 & $2.23 \pm 0.042^{* * *}$ & $2.11 \pm 0.006^{* \star *}$ & $7.31 \pm 0.030^{* * *}$ & $1.03 \pm 0.034^{* \star *}$ & 72.0 \\
\hline$X I X$ & $2 p$ & $5.38 \pm 0.040^{\star \star \star}$ & $3.81 \pm 0.065^{\star \star \star}$ & $12.11 \pm 0.040^{* \star \star}$ & $5.73 \pm 0.017^{\star}$ & 80.3 \\
\hline$X X$ & $2 q$ & $4.13 \pm 0.088^{* \star *}$ & $3.81 \pm 0.065^{* \star *}$ & $8.70 \pm 0.063^{* \star \star}$ & $2.45 \pm 0.143^{* \star *}$ & 74.0 \\
\hline$X X I$ & $2 r$ & $4.83 \pm 0.034^{* * *}$ & $4.91 \pm 0.054^{* * *}$ & $10.46 \pm 0.147^{* \star \star}$ & $4.36 \pm 0.084^{* * *}$ & 89.0 \\
\hline XXII & $2 s$ & $4.50 \pm 0.051^{* \star *}$ & $4.33 \pm 0.666^{* * *}$ & $9.56 \pm 0.105^{* \star \star}$ & $4.41 \pm 0.130^{* * *}$ & 90.0 \\
\hline XXIII & $2 \mathrm{t}$ & $5.16 \pm 0.049^{* * *}$ & $4.81 \pm 0.054^{* * *}$ & $4.8 \pm 0.051^{* \star \star}$ & $4.63 \pm 0.135^{* \star *}$ & 72.0 \\
\hline
\end{tabular}

Values are expressed as mean \pm SEM for six animals; ${ }^{* *} p<0.05,{ }^{*} p<0.001$ compared to control group; phenytoin dose: 25 $\mathrm{mg} / \mathrm{kg}$; test: $30 \mathrm{mg} / \mathrm{kg}$

Table 2: Anticonvulsant activity of 2-(Substituted)-N- o-tolyl-5,6-dihydro-4H-cyclopenta[b]thiophene-3carboxamide on pentylene tetrazole-induced convulsions (PTZ)

\begin{tabular}{|c|c|c|c|c|c|}
\hline Group & Treatment & $\begin{array}{l}\text { Onset of clonic } \\
\text { convulsion (s) }\end{array}$ & $\begin{array}{l}\text { Onset of tonic } \\
\text { convulsion (s) }\end{array}$ & $\begin{array}{c}\% \text { Protection after } \\
30 \mathrm{~min}\end{array}$ & $\begin{array}{c}\% \text { Protection after } \\
24 \mathrm{~h} \\
\end{array}$ \\
\hline I & Control & $47.1 \pm 0.108$ & $6.21 \pm 0.040$ & 0 & 0 \\
\hline II & Diazepam & $0.00 \pm 0.000$ & $0.00 \pm 0.000$ & 100 & 100 \\
\hline III & 2 & $17.4 \pm 0.084^{* * *}$ & $5.72 \pm 0.007^{*}$ & 66.66 & 33.33 \\
\hline IV & $2 a$ & $15.1 \pm 0.036^{* * *}$ & $4.88 \pm 0.007^{* * t}$ & 50 & 16.66 \\
\hline V & $2 b$ & $10.2 \pm 0.030^{* * *}$ & $2.07 \pm 0.002^{* * *}$ & 83.33 & 83.33 \\
\hline $\mathrm{VI}$ & $2 c$ & $10.4 \pm 0.066^{* * *}$ & $1.83 \pm 0.049^{* * *}$ & 33.33 & 16.66 \\
\hline VII & $2 d$ & $12.2 \pm 0.016^{* * *}$ & $1.23 \pm 0.080^{* * *}$ & 83.33 & 83.33 \\
\hline VIII & $2 e$ & $13.8 \pm 0.018^{* * *}$ & $1.36 \pm 0.088^{* * * t}$ & 83.33 & 50 \\
\hline IX & $2 f$ & $12.0 \pm 0.033^{* * *}$ & $1.03 \pm 0.009^{* * t}$ & 83.33 & 83.33 \\
\hline$x$ & $2 g$ & $11.2 \pm 0.006^{* * *}$ & $1.31 \pm 0.098^{* * *}$ & 66.66 & 50 \\
\hline$X I$ & $2 \mathrm{~h}$ & $16.8 \pm 0.015^{* * *}$ & $1.53 \pm 0.111^{* * * *}$ & 66.66 & 66.66 \\
\hline XII & $2 i$ & $14.4 \pm 0.026^{* * *}$ & $1.48 \pm 0.065^{* * *}$ & 83.33 & 50 \\
\hline XIII & $2 \mathrm{j}$ & $11.1 \pm 0.005^{* * *}$ & $1.23 \pm 0.084^{* * *}$ & 66.66 & 33.33 \\
\hline XIV & $2 k$ & $12.0 \pm 0.022^{* * * t}$ & $1.31 \pm 0.107^{* * *}$ & 83.33 & 66.66 \\
\hline$X V$ & 21 & $14.8 \pm 0.004^{* * *}$ & $1.85 \pm 0.194^{* * *}$ & 66.66 & 50 \\
\hline $\mathrm{XVI}$ & $2 m$ & $9.98 \pm 0.009^{* * *}$ & $0.95 \pm 0.011^{* * *}$ & 83.33 & 83.33 \\
\hline$X V I I$ & $2 n$ & $10.9 \pm 0.020^{* * *}$ & $0.96 \pm 0.014^{* * *}$ & 83.33 & 66.66 \\
\hline XVIII & 20 & $10.1 \pm 0.004^{* * *}$ & $1.03 \pm 0.034^{* * *}$ & 50 & 50 \\
\hline XIX & $2 p$ & $24.2 \pm 0.002^{* * *}$ & $5.73 \pm 0.017^{*}$ & 50 & 50 \\
\hline$x x$ & $2 q$ & $9.83 \pm 0.168^{* * *}$ & $2.45 \pm 0.143^{* * *}$ & 83.33 & 83.33 \\
\hline$X X I$ & $2 r$ & $18.4 \pm 0.028^{* * *}$ & $4.36 \pm 0.084^{* * *}$ & 83.33 & 83.33 \\
\hline XXII & $2 s$ & $16.8 \pm 0.080^{* * *}$ & $4.41 \pm 0.130^{* * *}$ & 83.33 & 66.66 \\
\hline
\end{tabular}

Values are expressed as mean \pm SEM for six animals; ${ }^{* \star *} p<0.05,{ }^{*} p<0.001$ compared to control group; diazepam dose: 5 $\mathrm{mg} / \mathrm{kg}$; dose of test compound: $30 \mathrm{mg} / \mathrm{kg}$ 
Table 3: ADME (absorption, distribution, metabolism, excretion) properties of 2-(Substituted)-N- otolyl-5,6-dihydro-4H-cyclopenta[b]thiophene-3-carboxamide

\begin{tabular}{|c|c|c|c|c|c|c|c|c|c|c|}
\hline Compound & CNS & $\begin{array}{c}\text { QPlogP } \\
\mathrm{po} / \mathrm{w}\end{array}$ & QPlogS & $\begin{array}{l}\text { Qplog } \\
\text { HERG }\end{array}$ & $\begin{array}{l}\text { QPP } \\
\text { Cacco }\end{array}$ & $\begin{array}{l}\text { Qplog } \\
\text { BB }\end{array}$ & $\begin{array}{c}\text { QPP } \\
\text { MDCK }\end{array}$ & $\begin{array}{l}\text { Qplog } \\
\text { Kp }\end{array}$ & $\begin{array}{l}\text { Qlog } \\
\text { Khsa }\end{array}$ & $\begin{array}{c}\text { \%Human } \\
\text { oral } \\
\text { absorption }\end{array}$ \\
\hline 2 & 0 & 3.443 & -4.338 & -4.637 & 1815.3 & -0.196 & 1726.854 & -2.017 & 0.33 & 100 \\
\hline $2 a$ & 1 & 5.367 & -6.001 & -5.886 & 4539 & 0 & 3870.398 & -0.487 & 0.882 & 100 \\
\hline $2 b$ & 0 & 4.929 & -5.902 & -5.814 & 1676.2 & -0.572 & 1349.691 & -1.299 & 0.749 & 100 \\
\hline $2 c$ & 0 & 5.72 & -6.739 & -5.963 & 4529.9 & -0.016 & 3898.422 & -0.675 & 1.058 & 100 \\
\hline $2 d$ & -1 & 4.495 & -4.84 & -5.455 & 1730.7 & -0.588 & 1251.683 & -1.101 & 0.531 & 100 \\
\hline $2 e$ & -1 & 4.341 & -5.162 & -5.547 & 735.63 & -0.963 & 547.676 & -1.907 & 0.575 & 100 \\
\hline $2 f$ & -2 & 4.311 & -5.769 & -6.004 & 442.98 & -1.289 & 317.55 & -2.372 & 0.624 & 100 \\
\hline $2 g$ & 0 & 6.351 & -7.415 & -5.979 & 4539.5 & -0.086 & 3865.438 & -0.672 & 1.303 & 100 \\
\hline $2 \mathrm{~h}$ & 1 & 5.659 & -6.394 & -5.782 & 4912.1 & 0.15 & 7718.86 & -0.542 & 0.935 & 100 \\
\hline $2 i$ & 0 & 5.801 & -6.879 & -5.965 & 4418.4 & -0.096 & 3773.591 & -0.686 & 1.044 & 100 \\
\hline $2 j$ & 1 & 5.973 & -6.512 & -5.066 & 4798 & 0.321 & 10000 & -0.816 & 1.004 & 100 \\
\hline $2 k$ & 1 & 5.611 & -6.131 & -5.625 & 4608.2 & 0.067 & 5134.292 & -0.542 & 0.962 & 100 \\
\hline 21 & 0 & 5.389 & -5.706 & -5.311 & 4700.1 & -0.106 & 3831.542 & -0.558 & 0.821 & 100 \\
\hline $2 m$ & 1 & 4.917 & -5.522 & -5.797 & 4991.1 & 0.056 & 4574.05 & -0.449 & 0.663 & 100 \\
\hline $2 n$ & 0 & 5.516 & -6.172 & -5.806 & 3237.3 & -0.147 & 2593.073 & -0.743 & 0.992 & 100 \\
\hline 20 & 0 & 4.763 & -6.15 & -5.993 & 1163.5 & -0.594 & 1028.937 & -1.674 & 0.779 & 100 \\
\hline $2 p$ & 0 & 5.458 & -6.415 & -5.031 & 3507.7 & -0.109 & 3011.975 & -1.346 & 1.045 & 100 \\
\hline $2 q$ & 1 & 3.974 & -4.281 & -5.782 & 1086.2 & 0.383 & 933.207 & -3.219 & 0.608 & 100 \\
\hline $2 r$ & 0 & 5.154 & -5.723 & -4.882 & 4853.3 & -0.015 & 4383.093 & -0.991 & 0.827 & 100 \\
\hline $2 s$ & 1 & 5.663 & -6.216 & -5.688 & 5253.6 & 0.063 & 4417.94 & -0.451 & 1.007 & 100 \\
\hline $2 t$ & 0 & 5.099 & -5.772 & -4.838 & 2927.7 & -0.201 & 2878.586 & -1.363 & 0.83 & 100 \\
\hline
\end{tabular}

Table 4: Docking studies of 2-(Substituted)-N- o-tolyl-5,6-dihydro-4H-cyclopenta[b]thiophene-3carboxamide

\begin{tabular}{|c|c|c|c|c|c|}
\hline Compound & $\begin{array}{l}\text { Dock } \\
\text { score }\end{array}$ & e model & $\begin{array}{l}\text { No of } \\
\text { bonds }\end{array}$ & $\begin{array}{l}\text { Interacted protein } \\
\text { residues }\end{array}$ & $\mathrm{H}$-bond distance \\
\hline 2 & -4.017 & -36.112 & 2 & Leu 130,Thr 132 & $1.825,2.017$ \\
\hline $2 a$ & -3.288 & -35.699 & 1 & Tyr 348 & 2.101 \\
\hline $2 b$ & -7.01 & -40.382 & 2 & Ile 350, Tyr 348 & $1.710,1.944$ \\
\hline $2 c$ & -4.341 & -33.889 & - & - & - \\
\hline $2 d$ & -2.2981 & -47.413 & 1 & Ile 350 & 2.176 \\
\hline $2 e$ & -6.121 & -42.201 & 4 & $\begin{array}{c}\text { Arg 343,Pro } 344, \text { Tyr } \\
348\end{array}$ & $\begin{array}{c}1.897,2.445,1.719,2.12 \\
2\end{array}$ \\
\hline $2 f$ & -6.865 & -45.184 & 4 & $\begin{array}{l}\text { Arg343,Pro } \\
\text { 344,Tyr348 }\end{array}$ & $\begin{array}{c}1.895,2.212,1.719,2.29 \\
0\end{array}$ \\
\hline $2 g$ & -4.186 & -42.744 & - & - & - \\
\hline $2 h$ & -4.425 & -28.5822 & 1 & Tyr 348 & 2.165 \\
\hline $2 i$ & -4.16 & -39.696 & 1 & Tyr 348 & 2.438 \\
\hline $2 \mathrm{j}$ & -4.076 & -37.851 & 1 & Tyr 348 & 2.118 \\
\hline $2 \mathrm{k}$ & -4.777 & -29.547 & 1 & Tyr 348 & 2.137 \\
\hline 21 & -4.311 & -36.503 & 1 & Tyr348 & 2.386 \\
\hline $2 m$ & -3.574 & -36.763 & - & - & - \\
\hline $2 n$ & 4.398 & -44.376 & - & - & - \\
\hline 20 & -5.136 & -54.835 & 1 & Ile 350 & 1.947 \\
\hline $2 p$ & -4.353 & -38.168 & 1 & Tyr 348 & 2.077 \\
\hline $2 q$ & -5.878 & -26.655 & 2 & Tyr 348,Gly104 & $2.162,2.328$ \\
\hline $2 r$ & -3.457 & -41.757 & 2 & Gly104,Leu 103 & $1.827,2.080$ \\
\hline $2 s$ & -3.462 & -37.648 & - & - & - \\
\hline $2 \mathrm{t}$ & -5.636 & -41.595 & 1 & Tyr 132 & 1.917 \\
\hline
\end{tabular}

'- indicates compound did not interacting with protein residues that had not hydrogen bond

peak and formation of $\mathrm{N}=\mathrm{CH}$ at $1569 \mathrm{Cm}^{-1}$ which means that the compound is derivatized. thiophene-3-carboxamide was treated with different aldehydes to form Schiff bases, and the IR spectra showed the dissappearance of $\mathrm{NH}_{2}$. 
Inhibition of MES test indicates activity against generalized tonic-clonic (grand mal) and cortical focal seizures [19]. It permits evaluation of the capacity of a substance to prevent seizure spread through neural tissue. MES test is not suitable for the testing of drugs acting on $\mathrm{Na}^{+}$ channels; the model is suitable for the evaluation of the majority of standard and newly developed anticonvulsant drugs, despite the fact that these drugs interact with other drug targets. In MESinduced convulsion model, reference drug, phenytoin $(25 \mathrm{mg} / \mathrm{kg})$ prevented the important tonic extensor phase of the tonic-clonc seizures, offered $100 \%$ protection and produced tonic flexion convulsions, whereas compounds $\mathbf{2 b}, \mathbf{2 e}$, 2f, 2m, 2n, 2o, 2q altered extensor phase duration and onset of clonus phase; probably due to the inhibition of high frequency action potential firing but the compounds did not totally abolish the various phases at $30 \mathrm{mg} / \mathrm{kg}$ dose.

PTZ is a most frequently used substance as well as an acute experimental model in the preliminary screening to test potential anticonvulsant drugs. PTZ is a selective blocker of the chloride ionophore complexation to the $\mathrm{GABA}_{\mathrm{A}}$ receptor, and after repeated or singledose administration, leads to a decrease in GABAergic function and to the stimulation and modification of density or sensitivity of different glutamate receptor subtypes in many brain regions [19]. The precise mechanism of the synthesized compounds is not clear, but in silico studies revealed that the action of the test substances was on the GABA mediated chloride channel.

\section{CONCLUSION}

A simple rapid, efficient synthesis of 2aminothiophenes by introduction of amide linkage at $3^{\text {rd }}$ position has been achieved by onepot synthesis utilizing cost-effective guanidinebased ionic liquid. Recent advances in the synthesis of 2-aminothiophenes reveal imidazoles-based ILs is replaced by guanidinebased ionic liquid. Isolation is simple, without the need for chromatographic purification, and there is no loss of catalytic activity. The anticonvulsant activity of the synthesized compounds is moderate against screening models. The results of in silco studies reveal significant role of topological parameters in inhibiting GABA-AT while docking results reveal significant role of phenyl ring and terminal carboxamide in drugreceptor interaction.

\section{REFERENCES}

1. Maloy KP, Gautam P, Vinita C, Manju YK, Sudhir S. Thiophenes containing triarylmethanes as
Antitubercular agents. Bioorg Med Chem Lett 2008; 18: 289-292.

2. Ferreira ICF, Calheha RC, Estevinho LM, Queiroz MJR. Screening of antimicrobial activity of diarylamines in the 2,3,5-trimethylbenzo[b]thiophenes series:A structure activity evaluation study. Bioorg Med Chem Lett 2004; 14: 5831-5833.

3. Bonini $C$, Chiummiento $L$, Bonis $M D$, Funicello $M$, Lupattelli $P$, Suanno G, Berti F,Campaner $P$. Synthesis,biological activity and modeling studies of two novel anti HIV PR inhibitors with a thiophene containing hydroxyethylamino core. Tetrahedron 2005; 61: 6580-6583.

4. Haider B, Hamada MI, Saad M, Mohamed H, Elnagdi, Huda M. 2-aminothiophenes as building blocks in heterocyclic synthesis:synthesis and antimicrobial evaluation of a new class of pyrido[1,2a]thieno[3,2-e]pyrimidine quinoline and pyridin-2one derivatives. European J Med Chem 2012; 52: 51-65.

5. Barnes DM, Haight AR, Hameury $T$, Maureen $A$, McLaughlin J, Mei J,Jason S, Tedrow JS, Toma JDR.New conditions for the synthesis of thiophenes via knoevenagal/Gewald reaction sequence.Application to the synthesis of multitargeted kinase inhibitor. Tetrahedron 2006; 62: 11311-11319.

6. Pier GB, Romeo R, Maria GP, Maria del CN, Mojgan $A T$, John C, Shrycok, Edward L,Allan R, Moorman "et al." synthesis and biological effect of novel 2amino-3-napthoylthiophenes as allosteric enhancers of the $A_{1}$ Adenosine receptor. J Med Chem 2003; 46: 797-809.

7. Stephanie H, Enrico P, Gilbert K. Microwave-assisted synthesis of 2-aminothiophene-3-carboxylic acid derivatives 3H-thieno[2,3-d]pyrimidin-4-one. Tetrahedron lett 2007; 48:5261-5264.

8. Firouz MM, Hassan ZB. A versatile one-pot synthesis of 2,3,5-tri-substituted thiophenes from thiomorpholides. Tetrahedron lett 2003; 44: 62536255.

9. Olaf Zeika, Horst $\mathrm{H}$. on the oxidative coupling of $\mathrm{N}, \mathrm{N}$ disubstituted 2-aminothiophenes-synthesis of N,N'persubstituted 5,5'-diamino-2,2'-bithiophenes Tetrahedron 2004; 60: 8213-8219.

10. Georgette M, Castanedo, Daniel PS. Synthesis of tetrasubstituted thiophenes on solid support using the Gewald reaction.Tetrahedron lett 2001; 42: 7181-7184.

11. Pushyamitra M, Hardesh K, Maurya, Brijesh K, Vishnu $K$, Tandon, Vishnu JR. synthesis of thiophenes and pyranone fused thiophenes by base induced inter and intra molecular C-S, C-C bond formation:a non catalytic approach. Tetrahedron lett $2012 ; 53: 1056$ 1059.

12. Bruno Dias DCF,Dos Santos, Joseu S, Bello F, Erika M, Carvolho D,Joel Jones J, Flavia MDS. A new protocol for the synthesis of 2-aminothiophenes through the Gewald reaction under solvent freeconditions .Hetero lett 2012; 2: 31-36.

13. Asghar D, Maryam I.Docking and QSAR studies of $\beta$ phenylethylidene hydrazine derivatives as a Gammaaminobutyric acid aminotransferase inhibitor. Med Chem Res 2011; 20: 955-961.

14. Acute oral toxicity-up- and- down procedure (UDP). OECD guidelines for testing of chemicals: 425. Adopted $3^{\text {rd }}$ October 2008, [2011 Oct 26]. Available from http://iccvam.niehs.nih.gov/SuppDocs/FedDoc s/OECD/OECDtg425.

15. Krall RL, Penry JK, White BG, Kupferberg HJ, Swinyard EA. Antiepileptic drug development. II: Anticonvuls Drug Screen. Epilepsia 1978; 19(4): 409-428.

16. Friesner RA, Banks JL, Murphy RB, Halgren TA, Klicic JJ, Mainz DT,Repasky MP, Knoll EH, Shaw DE, Shelley M, Perry JK, Francis P, Shenkin,PS Glide. 
A New Approach for Rapid, Accurate Docking and Scoring. 1. Method and Assessment of Docking Accuracy, J. Med. Chem. 2004; 47: 1739-1749.

17. Halgren TA, Murphy RB, Friesner RA, Beard HS, Frye LL, Pollard WT, Banks JL. Glide: A New Approach for Rapid, Accurate Docking and Scoring. 2. Enrichment Factors in Database Screening. J. Med. Chem. 2004; 47: 1750-1759.

18. Friesner RA, Murphy RB. Repasky MP, Frye $L L$, Greenwood JR, Halgren, TA., Sanschagrin, $P C$ Mainz DT. Extra Precision Glide: Docking and Scoring Incorporatinga Model of Hydrophobic
Enclosure for Protein-Ligand Complexes. J. Med. Chem. 2006; 49: 6177-6196.

19. Shirish D, Ambawade VS, Kasture SB. Anticonvulsant activity of roots and rhizomes of Glycyrrhiza glabra. Indian J Pharmacol 2002: 34:251-255.

20. Arbrar MT, Rukshana AR, Pinaki Ghosh, Bodhankar SL.Antiepileptic activity of lobeline isolated from the leaf of Lobelia nicotianaefolia and its effect of brain GABA level in mice. Asian Pacific J Trop Biomed 2012; 2: 537-542. 